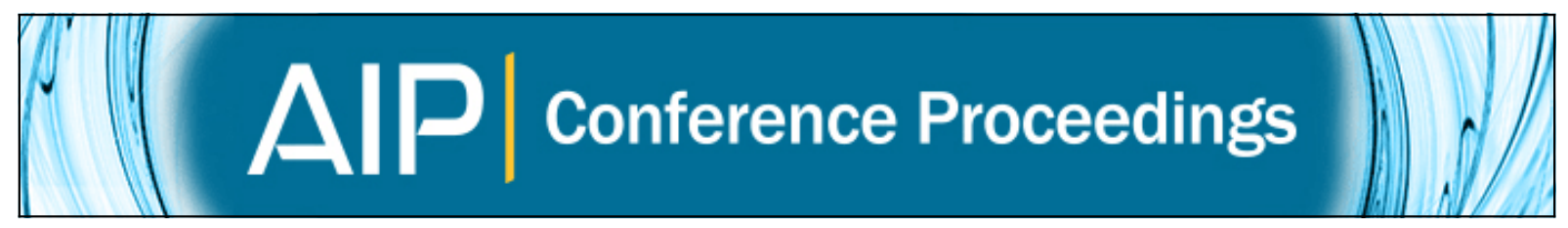

\title{
SuperCDMS Detector Fabrication Advances
}

P. L. Brink, Z. Ahmed, D. S. Akerib, C. N. Bailey, D. Balakishiyeva, D.A. Bauer, J. Beaty, R. Bunker, B. Cabrera,

D. O. Caldwell, J. Cooley, E. do Couto e Silva, P. Cushman, M. Daal, F. DeJongh, M. R. Dragowsky, L. Duong,

E. Figueroa-Feliciano, J. Filippini, M. Fritts, S. R. Golwala, D. R. Grant, J. Hall, R. Hennings-Yeomans, S. Hertel,

A. Hojem, D. Holmgren, L. Hsu, M. E. Huber, K. D. Irwin, O. Kamaev, M. Kos, M. Kiveni, S. W. Leman, R.

Mahapatra, V. Mandic, K. A. McCarthy, D. Moore, N. Mirabolfathi, H. Nelson, L. Novak, R. W. Ogburn, M. Pyle,

X. Qiu, E. Ramberg, W. Rau, A. Reisetter, T. Saab, B. Sadoulet, J. Sander, R. Schmitt, R. W. Schnee, D. N.

Seitz, B. Serfass, K. M. Sundqvist, A. Tomada, G. Wang, P. Wikus, S. Yellin, J. Yoo, and B. A. Young

Citation: AIP Conference Proceedings 1185, 655 (2009); doi: 10.1063/1.3292427

View online: http://dx.doi.org/10.1063/1.3292427

View Table of Contents: http://scitation.aip.org/content/aip/proceeding/aipcp/1185?ver=pdfcov

Published by the AIP Publishing

\section{Articles you may be interested in}

Background considerations for SuperCDMS

AIP Conf. Proc. 1549, 223 (2013); 10.1063/1.4818113

Characterization of SuperCDMS 1-inch Ge Detectors

AIP Conf. Proc. 1185, 659 (2009); 10.1063/1.3292428

SuperCDMS Detector Readout Cryogenic Hardware

AIP Conf. Proc. 1185, 282 (2009); 10.1063/1.3292334

A Measurement of Electron and Hole Drift Velocities in a Germanium CDMS Detector, at a Temperature of 31 milliKelvin

AIP Conf. Proc. 1185, 128 (2009); 10.1063/1.3292299

Status and future of the CDMS experiment: CDMS-II to SuperCDMS

AIP Conf. Proc. 957, 193 (2007); 10.1063/1.2823758 


\title{
SuperCDMS Detector Fabrication Advances
}

\author{
P. L. Brink ${ }^{\mathrm{a}}$, Z. Ahmed ${ }^{\mathrm{b}}$, D. S. Akerib ${ }^{\mathrm{c}}$, C. N. Bailey ${ }^{\mathrm{c}}$, D. Balakishiyeva ${ }^{\mathrm{e}}$,
} D. A. Bauer ${ }^{\mathrm{d}}$, J. Beaty $^{\mathrm{p}}$, R. Bunker ${ }^{\mathrm{m}}$, B. Cabrera ${ }^{\mathrm{a}}$, D. O. Caldwell ${ }^{\mathrm{m}}$, J. Cooley $^{\mathrm{a}}$, E. do Couto e Silva ${ }^{i}$, P. Cushman ${ }^{p}$, M. Daal ${ }^{1}$, F. DeJongh ${ }^{d}$, M. R. Dragowsky ${ }^{c}$, L. Duong ${ }^{\mathrm{p}}$, E. Figueroa-Feliciano ${ }^{\mathrm{e}}$, J. Filippini ${ }^{\mathrm{b}}$, M. Fritts ${ }^{\mathrm{p}}$, S. R. Golwala ${ }^{\mathrm{b}}$, D. R. Grant ${ }^{\mathrm{c}}$, J. Hall ${ }^{\mathrm{e}}$, R. Hennings-Yeomans ${ }^{\mathrm{c}}, \mathrm{S}$. Hertel ${ }^{\mathrm{e}}$, A. Hojem ${ }^{\mathrm{c}}$,

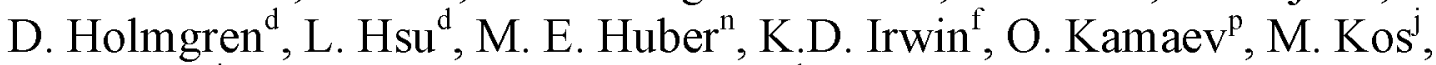
M. Kiveni ${ }^{j}$, S.W. Leman ${ }^{\mathrm{e}}$, R. Mahapatra ${ }^{\mathrm{k}}$, V. Mandic ${ }^{\mathrm{p}}$, K. A. McCarthy ${ }^{\mathrm{e}}$,

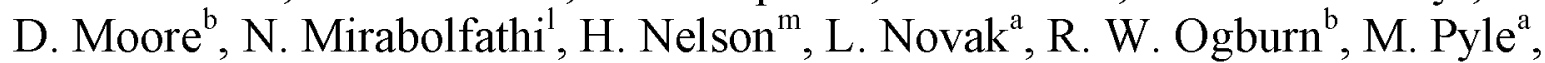

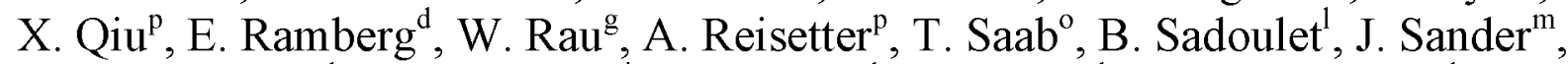

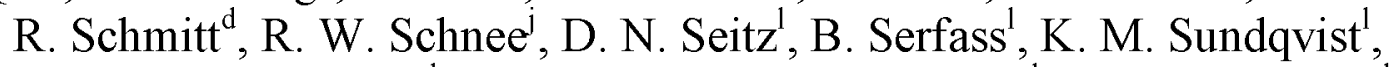

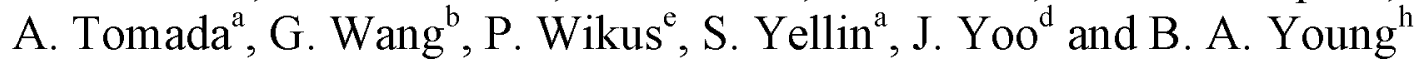

${ }^{a}$ Department of Physics, Stanford University, Stanford CA 94305, USA.

${ }^{b}$ Department of Physics, California Institute of Technology, Pasadena, CA 91125, USA.

${ }^{c}$ Department of Physics, Case Western Reserve University, Cleveland, OH 44106, USA.

${ }^{d}$ Fermi National Accelerator Laboratory, Batavia, IL 60510, USA.

¿Department of Physics, MIT, Cambridge, MA 02139, USA.

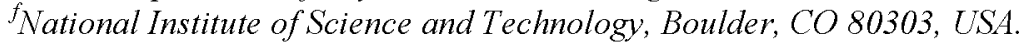

${ }^{g}$ Department of Physics, Queens' University, Kingston, Ontario, K7L 3N6, Canada.

${ }^{h}$ Department of Physics, Santa Clara University, Santa Clara, CA 95053, USA.

${ }^{i} S L A C$ National Accelerator Laboratory/KIPAC, Menlo Park, CA 94025, USA.

${ }^{j}$ Department of Physics, Syracuse University, Syracuse, NY 13244, USA.

${ }^{k}$ Department of Physics, Texas A\&M University, College Station, TX 77843 USA.

${ }^{l}$ Department of Physics, University of California, Berkeley, CA 94720, USA.

${ }^{m}$ Department of Physics, University of California, Santa Barbara, CA 93106, USA.

${ }^{n}$ Deptartments of Phys. \& Elec. Engr., University of Colorado, Denver, CO 80217, USA.

${ }^{\circ}$ Department of Physics, University of Florida, Gainesville, FL 32611, USA.

${ }^{p}$ School of Physics \& Astronomy, University of Minnesota, Minneapolis, MN 55455.

\begin{abstract}
For its dark matter search the SuperCDMS collaboration has developed new Ge detectors using the same athermal phonon sensors and ionization measurement technology of CDMS II but with larger mass, superior sensor performance and increased fabrication efficiency. The improvements in fabrication are described, a comparison of CDMS II and SuperCDMS detector production yield is reported, and future scalability addressed.
\end{abstract}

Keywords: Dark Matter. Cryogenic detectors. Transition Edge Sensors. Semiconductor fabrication. PACS: 14.80 Ly $95.35+85.25 . \mathrm{Oj}$

\section{INTRODUCTION}

Other papers in these proceedings [1-4] discuss various aspects of the detector performance advances that have occurred in the CDMS detectors [5]. The migration from the $10 \mathrm{~mm}$ thick substrates used for CDMS II [5] to the $25 \mathrm{~mm}$ thick substrates for the initial phases of the SuperCDMS program [6] facilitated three objectives: increase detector fabrication and testing throughput; reduction in surface 
contamination event rate by the increase in volume-tosurface ratio; and provide opportunities for exploring more advanced sensor designs [4]. Here we will focus on the logistics of the new detector fabrication program undertaken at the Stanford Nanofabrication Facility, the improvements since CDMS II, and production prospects for the future.

\section{SUPERCDMS DETECTOR FABRICATION FLOW CHART}

The increase in substrate thickness from the $10 \mathrm{~mm}$ of CDMS II to the $25 \mathrm{~mm}$ of SuperCDMS required new customized equipment for photoresist exposures (an EV Group model 620 contact aligner), photoresist coatings (Laurell), dry-etching (Drytek Corporation) and major modifications to our thin-film deposition machine (Balzers). This work was performed in stages, starting at the end of CDMS II fabrication in 2004 to entering production for SuperCDMS in 2008.
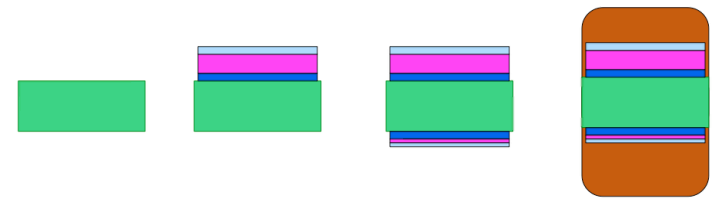

FIGURE 1. Initial steps in detector fabrication now used for SuperCDMS (geometry heavily distorted). First the 1-inch thick Ge substrates are cleaned (xylenes, acetone, isopropyl alcohol) and prepared (10\% HF dip for 8 minutes) prior to loading in a DC sputterer; then a trilayer of amorphous Si $(40 \mathrm{~nm}), \mathrm{Al}(300 \mathrm{~nm})$ and $\mathrm{W}(30 \mathrm{~nm})$ is deposited on the top side; followed by the bottom-side trilayer of amorphous $\mathrm{Si}$ $(40 \mathrm{~nm}), \mathrm{Al}(20 \mathrm{~nm})$ and $\mathrm{W}(20 \mathrm{~nm})$. After unloading, a 7 micron thick photoresist layer is applied to protect the bottom-set of films, followed by a 1.8-micron thick photoresist layer on the top-side to start the phonon sensor fin-layer patterning steps.
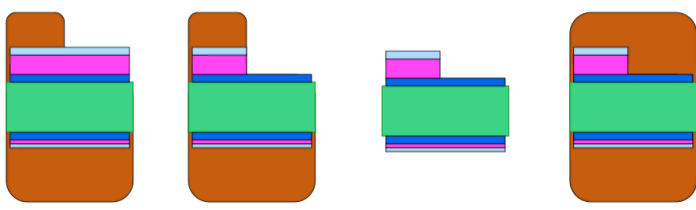

FIGURE 2. Top-side film patterning for the phonon collection fins: the top-side photoresist is exposed to the fin mask and developed; the exposed $\mathrm{W}$ and $\mathrm{Al}$ films are selectively wet-etched with $\mathrm{H}_{2} \mathrm{O}_{2}$ and commercial $\mathrm{Al}$ etchant, followed by a second etch with $\mathrm{H}_{2} \mathrm{O}_{2}$ to over-etch the $\mathrm{W}$ over-hang in order to improve step-coverage for the upcoming W TES film deposition. Both photoresist layers are chemically stripped; photoresist layers are reapplied but now the already-patterned fin-layer is protected with thick photoresist while the bottom-side is prepared for the ionization-grid electrode patterning
The fabrication flowchart established for SuperCDMS (see Figs. 1-5) was rearranged from that used for CDMS II [5] with the bottom-side grid now patterned before rather than after the Transition Edge Sensor (TES) layer. This change was needed both to reduce the degradation in the amorphous $\mathrm{Si}$ layer (stress-relieving by patterning the overlying high residual resistivity ratio but also highly compressive Al film) and to improve the reliability of the photolithographic procedure: the procedures depicted in Figs. 1,2 for the top-side fin layer and Figs. 2,3 for the bottom-side ionization grid layer are very similar to each other and use the same photoresist: Shipley 1813.

The ZIP (Z-dependent Ionization and Phonon) detectors of CDMS II require several hundred TESs electrically connected in parallel covering the surface of the detector surface (3-inch in diameter) as uniformly as possible to read out the phonon signal from the underlying substrate via the Al phonon collection fins [5]. In order to match readout impedance and operational criteria [7] the CDMS TESs need to be as narrow as possible. This requirement had a major effect on over-all detector yield (see Table 1).

For SuperCDMS it was no longer possible to use the stepper used for CDMS II with its 0.8 micron linewidth. Instead we relaxed the TES line-width to 1.6 microns and allowed the use of contact aligners. The accompanying phonon sensor redesign resulted in no degradation in performance [1]. The migration to a customized whole-field contact aligner aided considerably in both device fabrication (elimination of previous exposure errors in automated alignment; and exposure times per detector reduced from hours to minutes) and packaging (greater flexibility in placement of wire-bonding pads and bias rails to allow easier repairs; and detector packaging time reduced from weeks to days).
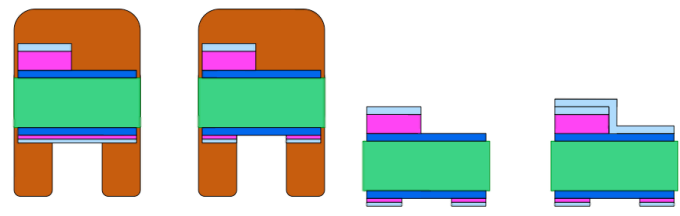

FIGURE 3. Bottom-side film patterning for the ionizationelectrode grid: the 1.6-micron thick photoresist is exposed to the grid mask and developed; the $\mathrm{W}$ and $\mathrm{Al}$ films are wetetched, but the amorphous Si film is left covering all the Ge substrate; both photoresist layers are chemically stripped; the detector receives a second $\mathrm{W}$ film deposition on the top side over the already-pattern fins that will become the transition edge sensors. 

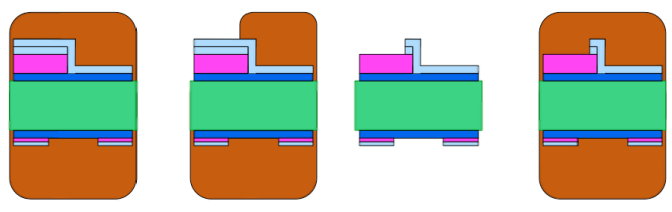

FIGURE 4. Top-side film patterning for the W TESs: a 7 micron thick photoresist layer protects the bottom-side ionization grid while a 1.0 -micron thick photoresist layer is applied to the top-side; the photoresist is exposed to the TES mask and developed; the exposed $\mathrm{W}$ is wet-etched; both photoresist layers are chemically stripped; photoresist layers are reapplied but now the already-patterned TES-layer is protected with thick photoresist while the bottom-side is prepared for the ionization electrode trench patterning.

The vulnerability of Shipley 3612 photoresist adhesion in hydrogen peroxide during the W TESs wet etch (see Fig. 4) was a major factor in the production yield for CDMS II (See Table 1 and discussion in following section). For SuperCDMS we developed an improved photoresist coating recipe where a preparation coating of AP-400 is applied to the $\mathrm{W}$ film before the high line-width-resolution 3612 photoresist is applied. This Adhesion Promoter (AP) improves the adhesion of the 3612 photoresist during the hydrogen peroxide etching and the fabrication yield for SuperCDMS was thus significantly improved.
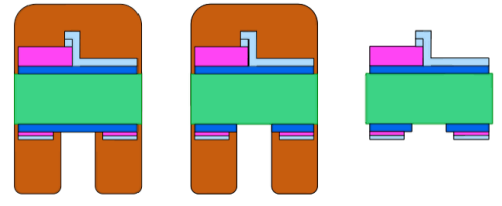

FIGURE 5. Bottom-side patterning of the amorphous $\mathrm{Si}$ layer to separate the inner and outer ionization electrode grids: the photoresist is exposed to the 'trench' mask and developed; the amorphous Si in the exposed trench is dryetched using $\mathrm{CHF}_{3}+\mathrm{O}_{2}$ (once exposed, the underlying Ge in the trench is etched to a depth of $600-900 \mathrm{~nm}$ ); both photoresist layers are chemically stripped and the detector is complete.

\section{COMPARISON OF CDMS II AND SUPERCDMS DETECTOR YIELD}

In Table 1 we summarize our progress through the detector tower deployments of CDMS II up to and including the first SuperTower of SuperCDMS that has recently been installed at the Soudan site. The quoted yields are rounded to the nearest $\%$.

The first category in Table 1, 'Crystal rejected' refers to both crystals damaged in initial polishing and crystals subsequently discovered in the testing program to suffer with ionization breakdown at relatively low applied ionization bias. Careful selection of vendors and revised specifications addressed these issues.

Both CDMS II and SuperCDMS candidate detectors can be rejected during the course of fabrication. Either severe photolithographic errors occur, equipment breaks down or the thin-films encounter stability, degradation, issues. Additionally during detector packaging detectors are rejected if excessive 'surgery' is needed to repair observable photolithographic defects.

The next column in Table 1, 'missing TESs', refers to the narrow TESs that could have been partially etched away during the W TES wet-etching (see Fig. 4). A dry-etch is not possible for this step as it would not be selective against the amorphous Si surrounding the TES. This class of visually 'border-line detectors' was a significant impacter on the acceptance yield at the CDMS-II testing facilities. Thus, part-way during CDMS II detector production, a liquid-nitrogen temperature vacuum cryostat was commissioned to identify such 'missing TESs' candidate detectors earlier; and also assess the Ge crystal quality with regard to possible leakage currents and ionization breakdown.

Moving onto the next two columns of Table 1, the W TES transition temperature $\left(T_{c}\right)$ and resistivity must be tightly controlled. If the $\mathrm{W} \mathrm{T}_{\mathrm{c}}$ is below $80 \mathrm{mK}$ then the detector cannot be operated. Alternatively, the $\mathrm{W}$ beta-phase could also occur with a high $\mathrm{W} \mathrm{T}_{\mathrm{c}}>180$ $\mathrm{mK}$ along-with a high normal-state resistivity.

TABLE 1. Overall detector yield on per-tower basis for CDMS II and the first SuperTower of SuperCDMS. See main text for column heading definitions. Each final tower for CDMS II contained six $10 \mathrm{~mm}$ thick substrates. For CDMS II Towers 1-5 the number of initial substrates used for each tower were 22, 18, 13, 16 and 13. For SuperCDMS there are five $25 \mathrm{~mm}$ thick Ge substrates per SuperTower [3] and the number of initial substrates for SuperTower 1 was 12.

\begin{tabular}{lccccccc}
\hline Tower deployed & $\begin{array}{c}\text { Crystal } \\
\text { rejected }\end{array}$ & $\begin{array}{c}\text { Abandoned in } \\
\text { fabrication }\end{array}$ & $\begin{array}{c}\text { Missing } \\
\text { TESs }\end{array}$ & $\begin{array}{c}\text { W Te } \\
\text { low }\end{array}$ & $\begin{array}{c}\text { W beta- } \\
\text { phase }\end{array}$ & $\begin{array}{c}\text { Sensor } \\
\text { rejected }\end{array}$ & Final Yield \\
\hline CDMS II Tower 1 & $32 \%$ & $5 \%$ & $5 \%$ & - & $4 \%$ & $27 \%$ & $27 \%$ \\
CDMS II Tower 2 & - & - & - & $50 \%$ & $11 \%$ & $6 \%$ & $33 \%$ \\
CDMS II Tower 3 & - & $23 \%$ & $8 \%$ & - & $23 \%$ & - & $46 \%$ \\
CDMS II Tower 4 & - & $25 \%$ & $25 \%$ & - & $13 \%$ & - & $37 \%$ \\
CDMS II Tower 5 & - & $15 \%$ & $38 \%$ & - & - & - & $47 \%$ \\
SuperCDMS Tower 1 & - & $42 \%$ & - & - & - & $17 \%$ & $41 \%$ \\
\hline
\end{tabular}


Mid-way during fabrication for CDMS II it had become clear that other users of the Balers sputtering machine could contaminate the machine and affect our $\mathrm{W}$ transition temperature. Thus stricter controls were implemented; in particular to prevent carbon contamination, which stabilized the growth of undesired beta-phase W. In addition an RGA system was installed to continuously monitor the chamber during sputtering. In 2004 the Balzers sputtering machine was moved to a new facility on the Stanford campus and came under our exclusive control.

Detectors were also rejected later in the CDMS II testing program. The 'sensor rejected' column in Table 1 includes: an unrepairable superconducting short in a phonon sensor, non-uniform detector response due to discontinuous regions within the phonon sensor (from surgery or film step coverage or degradation of amorphous-Si layer) or suppressed phonon signals due to low $\mathrm{W} \mathrm{T}_{\mathrm{c}}$ after ion implantation (from uncertainties in determining $\mathrm{T}_{c}$ map [8] distribution).

A final observation on the trends in Table 1 is to note that in the early phase of CDMS II detector fabrication was the production bottleneck and that cryogenic testing of all detector candidates was performed. More recently the decision bias has switched to rejecting questionable detectors as soon as possible. For Super-Tower 1, of the 12 initial Ge crystals, five detectors were rejected during fabrication leaving seven candidates for the cryogenic testing program. Two of the candidates were rejected during testing, giving an acceptance yield of $71 \%$. For SuperTower 2, whose testing program is underway, improvements in fabrication recipes and equipment refurbishment resulted in no candidate detectors being rejected during fabrication or packaging.

\section{FUTURE SCALABILITY}

An important requirement for future scalability of TES-based dark matter detectors is the control of the TES transition temperature, ideally it would not need cryogenic verification prior to assembly of the target. We have recently made significant progress in our continued use of $\mathrm{W}$ as future candidate material. The W metal target used for the last 7 years ran out in July 2008 and was replaced with a new target with a few ppm of iron impurity, an order of magnitude higher than the previous target. This reduced the $\mathrm{W} \mathrm{T}_{\mathrm{c}}$ from $\sim$ $140 \mathrm{mK}$ of CDMS II to $\sim 100 \mathrm{mK}$ with gradients of $<$ $5 \mathrm{mK}$ across 3 -inch diameter substrates. Since the starting $\mathrm{W} \mathrm{T}_{\mathrm{c}}$ is close to the desired operation value of $80 \mathrm{mK}$ any future ion-implantation of the $\mathrm{W}$ will be a lower, uniform, dose that will reduce the damage to the amorphous Si surrounding the TESs (see Ref [2] in these proceedings). To further improve uniformity we have modified the palettes that hold substrates in the sputtering machine to center the substrates with respect to the targets. This configuration is now in use and production for SuperTower 3 has commenced.

A restriction on scalability presently apparent in detector fabrication is the manually intensive multiple photoresist coating and baking regimes shown in Figs. $1-5$. We are exploring automation of this process using substrates held in boats and served by a robot arm between spinners and a stack of ovens. We believe such approaches performed at facilities available to the SuperCDMS collaboration members will bring the 1-2 orders of magnitude increase in fabrication throughput desired for future larger dark matter searches.

Our recent advances in control of the $W T_{c}$ has shifted our focus to reducing the occurrence of subKelvin superconducting shorts. We are in the process of commissioning a large He-3 cryostat with simplified wiring and readout electronics to screen multiple detectors for superconducting $\mathrm{Al}$ shorts.

Recent successes in the SuperCDMS detector fabrication program positions us well for the completion of the SuperCDMS Soudan project to install 5 SuperTowers at the CDMS Soudan site [3]. Plans are underway to expand and automate detector fabrication and testing to allow CDMS-style detectors to be competitive in future $100 \mathrm{~kg}$ and 1 tonne-scale dark matter search experiments.

\section{ACKNOWLEDGMENTS}

This work is supported in part by the National Science Foundation (Grant Nos. AST-9978911, PHY0542066, PHY-0503729, PHY-0503629, PHY0503641, PHY-0504224, PHY-0705052, PHY0801536, PHY-0801708, PHY-0801712 and PHY0802575 ), by the Department of Energy (Contracts DE-AC03-76SF00098, DE-FG02-91ER40688, DEFG03-90ER40569, DOE Contract DE-AC0276SFO0515 and DE-FG03-91ER40618), and by NSERC Canada (Grant SAPIN 341314-07).

\section{REFERENCES}

1. Z. Ahmed et al. these proceedings.

2. C.N. Bailey et al. these proceedings.

3. N. Mirabolfathi et al. these proceedings.

4. M. Pyle et al. these proceedings.

5. D.S. Akerib et al. (CDMS Collab.) Phys. Rev. D72, 052009 (2005).

6. D.S. Akerib et al. (SuperCDMS Collab.) J. Low Temp. Phys. 151 818-823 (2008).

7. B. Cabrera Nucl. Instrum. Methods A 444 304, (2000).

8. P.L. Brink et al. AIP Conference Proceedings, 605, 529 (2002). 